\title{
Low-dose HRTEM Study of Interstratified Clay Minerals
}

\author{
Guanyu Wang ${ }^{1,2}$, Hejing Wang ${ }^{2}$, Limin Zhang ${ }^{3,4}$, Hailiang Dong ${ }^{4}$, Kenneth M Kemner ${ }^{3}$ and Jianguo \\ Wen $^{1}$ \\ 1. Center for Nanoscale Materials, Argonne National Laboratory, Lemont, IL, USA. \\ 2. School of Earth and Space Sciences, Peking University, Beijing, China. \\ 3. Biosciences Division, Argonne National Laboratory, Lemont, IL, USA. \\ 4. State Key Laboratory of Biology and Environmental Geology, China University of Geosciences, \\ Beijing, China.
}

The abundantly present interstratified clay minerals are intermediate products of two or more end-member clays, which are widely documented in relation to weathering reactions. They provide valuable information of the formation and alteration nature of clay minerals [1]. Interstratification was first discovered and identified mainly by XRD in the past, however, it failed to provide individual crystal information as the tool averages millions of crystals. In the past 30 years, it is a great challenge to obtain high quality high-resolution transmission electron microscopy (HRTEM) images [2]. Viewing the interstratified clays requires edge on direction, which is hard to achieve due to the preferred orientation of such scaly mineral. Another challenge is the severe e-beam damage due to the existence of hydroxyl in clay structure [3]. In this study, special sample preparation method was used to obtain more edge on areas. Low-dose (below $\sim 5 \mathrm{e}^{-/} \AA^{2} \mathrm{~s}$ ) HRTEM imaging method with both spherical $\left(\mathrm{C}_{\mathrm{s}}\right)$ and chromatic aberration $\left(\mathrm{C}_{\mathrm{c}}\right)$ corrections were also applied. The structure of interstratified clays without interlayer ion, with $\mathrm{K}^{+}$as interlayer cation, with brucite-like layer, and with organics in the interlayer are captured.

Clay minerals are phyllosilicate with the basic structure of one octahedral layer (O layer, light blue sheets in Fig. 1, 2) sandwiched between two tetrahedral layers (T layer, yellow sheets in Fig. 1, 2). Talc and pyrophyllite are typical clay minerals without interlayer ion, whose average lattice spacing is $\sim 9.5 \AA$. Top of Fig. 1 shows such $\sim 9.5 \AA$ mineral structure interstratified with chlorite. With brucite-like layer (B layer, dark blue sheets in Fig. 1,2) in the interlayer, chlorite has an average lattice spacing of $\sim 14.3 \AA$, and the distance between $\mathrm{T}$ layer and the additional $\mathrm{B}$ layer is larger than that between $\mathrm{T}$ and $\mathrm{O}$ layer. Fig. 1 also shows $\sim 10 \AA$ mineral with $\mathrm{K}^{+}$as interlayer cation. The $\mathrm{T}$ layers and $\mathrm{K}^{+}$layer are bright dots while the $\mathrm{O} / \mathrm{B}$ layers are lines formed by continuous dots.

Fig. 2 shows high resolution images of interstratified clays. Fig. 2a shows chlorite $(\sim 14 \AA)$ interstratified with the $\sim 24 \AA$ clay, and the $\sim 24 \AA$ clay has the $14 \AA$ (TOTB) $+10 \AA$ (TOT) structure. Clay minerals, both in its natural and modified forms, are cheap materials successfully used as good adsorbent for the removal of toxic heavy metals and organic waste [4]. Fig. 2b shows the DFOB modified nontronite (originally $\sim 10 \AA$ ) intended for adsorption. After modification, the $\sim 5 \AA$ organic layer was inserted into the interlayer of nontronite [5].

References:

[1] J Środoń, Annual Review of Earth and Planetary Sciences 27(1) (1999), p. 19.

[2] S Lee and H Xu, Powder Diffraction. 32(2) (2017), p. 118.

[3] T Chen et al., American Mineralogist. 98(5-6) (2013), p. 879.

[4] MK Uddin, Chemical Engineering Journal. 308 (2017), p. 438. 
[5] Electron microscopy was performed at the Center for Nanoscale Materials at Argonne National Laboratory, a U.S. Department of Energy Office of Science Laboratory operated under Contract No. DE-AC02-06CH11357 by UChicago Argonne, LLC. This research is also supported by the National Natural Science Foundation of China (No 41872048) led by Hejing Wang. Guanyu Wang is grateful for the financial support from China Scholarship Council (No 201806010080) and technical support from Qingyun Li and Duan Luo.

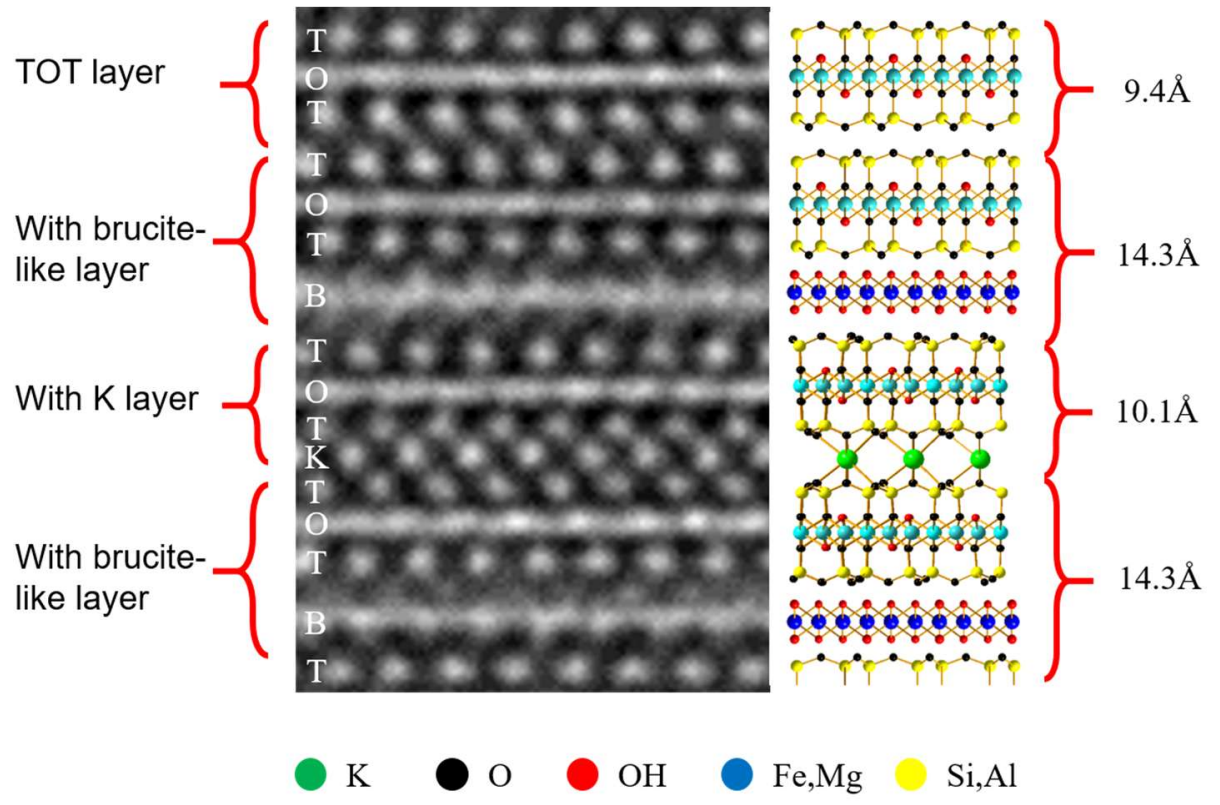

Figure 1. Low-dose HRTEM images of chlorite $(14.3 \AA)$ interstratified with $\sim 9.5 \AA$ and $\sim 10 \AA$ clay mineral. The $\sim 9.5 \AA$ clay has TOT structure without interlayer ion. The $\sim 10 \AA$ clay has $\mathrm{K}^{+}$as interlayer cation. The $14.3 \AA$ chlorite has a brucite-like layer in the interlayer.

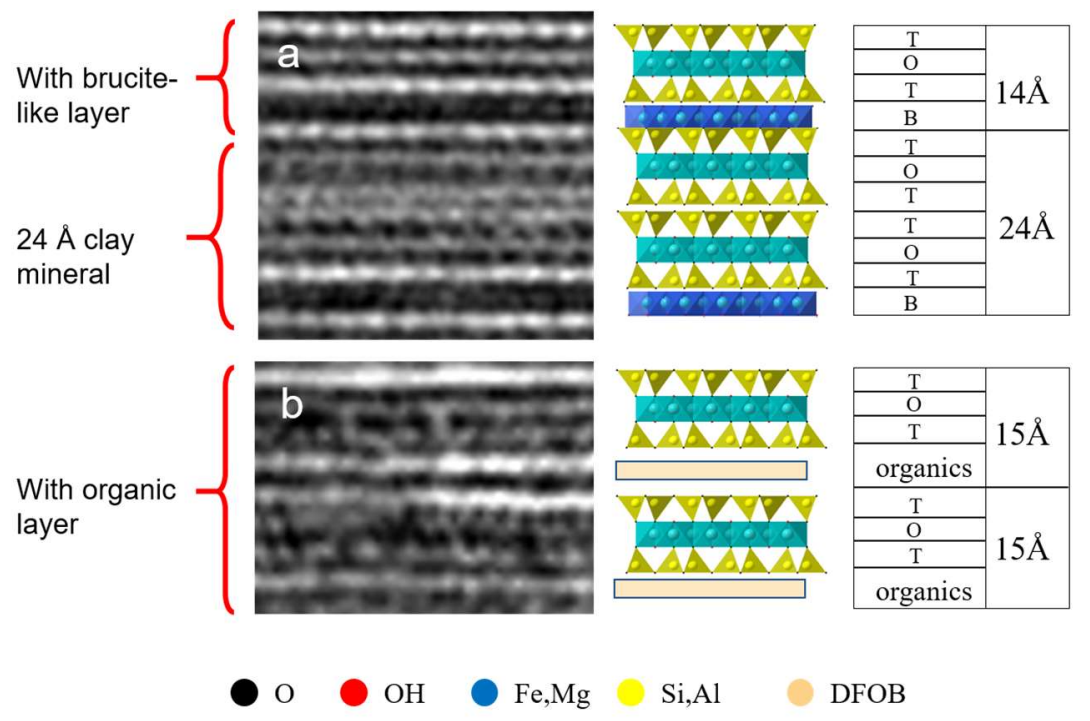

Figure 2. HRTEM images of a) chlorite $(\sim 14 \AA)$ interstratified with the $\sim 24 \AA$ clay mineral, and $b)$ the DFOB modified nontronite, the $\sim 5 \AA$ organic layer was inserted into the interlayer of nontronite (originally $\sim 10 \AA$ ). T refers to tetrahedral layers, marked as yellow sheets. O refers to octahedral layers, marked as light blue sheets. B refers brucite-like layers, marked as dark blue sheets. 